\title{
A CRITERION FOR SELF-SIMILAR SETS TO BE TOTALLY DISCONNECTED
}

\author{
Jun Luo and Dong Hong Xiong \\ Chongqing University, College of Mathematics and Statistics \\ 401331 Chongqing, P. R. China; jun.luo@cqu.edu.cn \\ Chongqing Business Vocational College, Department of Public Administration \\ Chongqing, 401331, P. R. China; 359570667@qq.com
}

\begin{abstract}
In this paper, we prove that, under Bandt's finite type condition, a self-similar set is totally disconnected if and only if the $\mathcal{E}$-connected families on every level are uniformly finite.
\end{abstract}

\section{Introduction}

Let $\left\{f_{i}\right\}_{i=1}^{m}$ be an iterated function system (IFS) on $\mathbf{R}^{d}$, where $f_{i}$ are contractive similitudes satisfying $\left|f_{i}(x)-f_{i}(y)\right|=r_{i}|x-y|$ for any $x, y \in \mathbf{R}^{d}$ and $0<r_{i}<1$ which are called contraction ratios. Hutchinson [6] proved that there exists a unique nonempty compact subset $K$ of $\mathbf{R}^{d}$ such that

$$
K=\bigcup_{i=1}^{m} f_{i}(K) .
$$

We call $K$ a self-similar set. By iteration, for each $k \geq 1$, we have $K=\bigcup_{\mathbf{i} \in \Sigma^{k}} f_{\mathbf{i}}(K)$ where $\Sigma=\{1, \ldots, m\}$ and $\mathbf{i}=i_{1} \cdots i_{k}$ means a finite word, $f_{\mathbf{i}}=f_{i_{1}} \cdots f_{i_{k}}$ a composition of maps. We write $K_{\mathbf{i}}=f_{\mathbf{i}}(K)$. When the contraction ratios are distinct, we need some modification to make the pieces $K_{\mathbf{i}}$ have approximately the same size. Let $r=\min _{i} r_{i}$. For $n \geq 1$, define the $n$-th level of finite words by

$$
\Lambda_{n}=\left\{\mathbf{i}=i_{1} \cdots i_{k}: r_{\mathbf{i}} \leq r^{n}<r_{i_{1}} \cdots r_{i_{k-1}}\right\} .
$$

Then the self-similar set can be written as

$$
K=\bigcup_{\mathbf{i} \in \Lambda_{n}} K_{\mathbf{i}}
$$

If a word $\mathbf{i} \in \Lambda_{n}$, we denote its length by $|\mathbf{i}|=n$. Let $W \subset \Lambda_{n}$, we call the family of sets $\left\{K_{\mathbf{i}}: \mathbf{i} \in W\right\}$ an $\mathcal{E}$-connected family if for any distinct $\mathbf{i}, \mathbf{j} \in W$, there exist $\mathbf{i}_{1}, \ldots, \mathbf{i}_{k} \in W$ satisfying $\mathbf{i}=\mathbf{i}_{1}, \mathbf{j}=\mathbf{i}_{k}$ and $K_{\mathbf{i}_{\ell}} \cap K_{\mathbf{i}_{\ell+1}} \neq \emptyset, \ell=1, \ldots, k-1$.

In 1985, Hata [5] first studied the structure of self-similar sets and showed that a self-similar set $K$ is connected if and only if $\left\{K_{i}: i \in \Sigma\right\}$ is an $\mathcal{E}$-connected family. Since then, the relevant topological questions on self-similar sets have attracted considerable attention. Many researches involve connectedness, local connectedness and disk-likeness of self-similar sets or self-affine tiles (see [8][3][11][12][10] and references therein).

https://doi.org/10.5186/aasfm.2021.4671

2020 Mathematics Subject Classification: Primary 28A80.

Key words: Totally disconnected, self-similar set, finite type, $\mathcal{E}$-connected family.

The research is supported by the Fundamental Research Funds for the Central Universities (Project No. 2019CDXYST0015) and Natural Science Foundation of Chongqing (No. cstc2019jcyjmsxmX0030). 
This short paper is about self-similar sets $K$ which are not connected, but may contain connected subsets. The following claim is well-known and easily proved:

If there is a connected subset $S$ with more than one point, then there are $\mathcal{E}$ connected families of arbitrary length.

Indeed, a minimal covering of $S$ by sets $f_{\mathbf{i}}(K)$ from $n$-th level must be $\mathcal{E}$ connected. The by triangle inequality, the sum of the diameters of the sets $f_{\mathbf{i}}(K)$ must be at least the diameter of $S$. Thus the number $N$ of the sets must fulfil $|S| \leq N r_{\max }^{n}|K|$ where $r_{\max }=\max _{i} r_{i}$ and $|E|$ denotes the diameter of the set $E$. Therefore, $N$ tends to infinity when $n$ goes to infinity.

However, the reverse of the claim was unknown. In this note we will prove that if Bandt's finite type condition (see the definition in Section 2) is fulfilled, then the reverse of the claim is true. There is a simple example showing that some spearation condition on the IFS is needed. Let $f_{1}(x)=x / 3, f_{2}(x)=x / 4$ and $f_{3}(x)=(x+4) / 5$ be an IFS on $\mathbf{R}$, then the self-similar set $K$ is totally disconnected since it has Hausdorff dimension strictly smaller than 1 . But we have arbitrarily large $\mathcal{E}$-connected families sharing one common point.

\section{Main result}

In order to analyse the topological structure of self-similar sets, Bandt and his collaborators [1,2] formulated a separation condition of finite type (here we call Bandt's finite type). Let $K$ be a self-similar set as in (1.1). If there are only finitely many neighbor maps $h:=f_{\mathbf{i}}^{-1} f_{\mathbf{j}}$ with $K \cap h(K) \neq \emptyset$ (or equivalently $f_{\mathbf{i}}(K) \cap f_{\mathbf{j}}(K) \neq$ $\emptyset)$ and with $\mathbf{i}, \mathbf{j} \in \Lambda_{n}$ for any $n \geq 1$, then the self-similar set $K$ is called to be of Bandt's finite type.

Bandt's finite type condition is a direct extension of Lagarias' condition [9] for patterns and tilings which states that a tile should have only finitely many neighborhoods of surrounding tiles. On the other hand, Ngai and Wang [13] have a similar finite type condition, which differs in Kenyon's example (see [7][4]) and in self-similar sets with incommensurable contraction ratios.

The following result was due to Bandt and Mesing [1], which is immediate from the definition. Here we provide a short proof for the sake of completeness.

Lemma 2.1. A self-similar set $K$ is of Bandt's finite type if and only if for any $R>0$,

$$
\#\left\{f_{\mathbf{i}}^{-1} f_{\mathbf{j}}: \mathbf{i}, \mathbf{j} \in \Lambda_{n}, n \geq 1, \text { and } f_{\mathbf{i}}^{-1} f_{\mathbf{j}}(K) \cap B(0, R) \neq \emptyset\right\}<\infty .
$$

Proof. The sufficiency is trivial since there exists a ball large enough such that $K \subset B(0, R)$. For the converse part, without loss of generality, we assume $0 \in K$. Let $\mathbf{i}, \mathbf{j} \in \Lambda_{n}$ and $h=f_{\mathbf{i}}^{-1} f_{\mathbf{j}}$ be a neighbor map of level $n$ if $K \cap h(K) \neq \emptyset$. The neighbor maps can be generated recursively from level $n$ to level $n+1$ in the sense that if $h=f_{\mathbf{i}}^{-1} f_{\mathbf{j}}$, then the neighbor maps of level $n+1$ for the pieces $K_{\mathbf{i i}_{1}}$ and $K_{\mathbf{j} \mathbf{j}_{1}}$ of $K_{\mathbf{i}}$ and $K_{\mathbf{j}}$, respectively, is given by

$$
g=f_{\mathbf{i}_{1}}^{-1} f_{\mathbf{i}}^{-1} f_{\mathbf{j}} f_{\mathbf{j}_{1}}=f_{\mathbf{i}_{1}}^{-1} h f_{\mathbf{j}_{1}},
$$

where $\mathbf{i}_{1}, \mathbf{j}_{1}$ are finite words such that $\mathbf{i i}_{1}, \mathbf{j j}_{1} \in \Lambda_{n+1}$. Hence we can obtain all neighbor maps in this way.

If $K \cap h(K)=\emptyset$, then the distance $d(K, h(K)):=\inf \{|x-y|: x \in K, y \in h(K)\}$ is positive, we set $d(K, h(K))>\epsilon>0$. For any $g=f_{\mathbf{i}_{1}}^{-1} h f_{\mathbf{j}_{1}}$, it follows that

$$
d(K, g(K))=d\left(f_{\mathbf{i}_{1}}^{-1}\left(K_{\mathbf{i}_{1}}\right), f_{\mathbf{i}_{1}}^{-1} h\left(K_{\mathbf{j}_{1}}\right)\right)=r_{\mathbf{i}_{1}}^{-1} d\left(K_{\mathbf{i}_{1}}, h\left(K_{\mathbf{j}_{1}}\right)\right) \geq \epsilon / r_{\mathbf{i}_{1}} .
$$


For any $R>0$, the distance can be larger than $R$ by several steps of generating, and $g(K) \cap B(0, R)=\emptyset$. That is, the number of neighbor maps $h$ satisfying both $h(K) \cap K=\emptyset$ and $h(K) \cap B(0, R) \neq \emptyset$ is finite. Suppose Bandt's finite type condition holds, then the number of neighbor maps $h$ satisfying both $h(K) \cap K \neq \emptyset$ and $h(K) \cap B(0, R) \neq \emptyset$ is finite as well. Consequently, the necessity follows.

Theorem 2.2. Suppose a self-similar set $K$ as in (1.1) is of Bandt's finite type. If $K$ is totally disconnected then the $\mathcal{E}$-connected families on every level are uniformly finite.

Proof. Let $R>3 r^{-1}|K|$ where $|K|$ denotes the diameter of $K$. We may assume the origin $0 \in K$. Let $\left\{g_{i}\right\}$ be the collection of maps of the form $f_{\mathbf{i}}^{-1} f_{\mathbf{j}}$ satisfying $g_{i}(K) \subset B(0, R)$ where $\mathbf{i}, \mathbf{j} \in \Lambda_{n}, n \geq 1$. Since $K$ is of Bandt's finite type, by Lemma 2.1, there are only finitely many of such maps, say, $g_{1}, \ldots, g_{N}$. Denote $F=\bigcup_{j=1}^{N} g_{i}(K)$, then $F \subset B(0, R)$. Note that if $K$ is totally disconnected, then so is $F$.

We first claim that: for $\mathbf{i}^{\prime} \in \Lambda_{p}, \mathbf{j} \in \Lambda_{n}$ with $p \leq n$, if $f_{\mathbf{i}^{\prime}}^{-1}\left(K_{\mathbf{j}}\right) \subset B\left(0,2 r^{-1}|K|\right)$ then $f_{\mathbf{i}^{\prime}}^{-1}\left(K_{\mathbf{j}}\right) \subset F$. Indeed, let $\mathbf{i}^{\prime}=\mathbf{i}_{1} \cdots \mathbf{i}_{p} \in \Lambda_{p}, \mathbf{j}=\mathbf{j}_{1} \cdots \mathbf{j}_{n} \in \Lambda_{n}$ where $p \leq n$. Clearly

$$
f_{\mathbf{i}^{\prime}}^{-1}\left(K_{\mathbf{j}}\right)=f_{\mathbf{i}_{1} \cdots \mathbf{i}_{p}}^{-1} f_{\mathbf{j}_{1} \cdots \mathbf{j}_{p}}\left(K_{\mathbf{j}_{p+1} \cdots \mathbf{j}_{n}}\right) \subset f_{\mathbf{i}_{1} \cdots \mathbf{i}_{p}}^{-1} f_{\mathbf{j}_{1} \cdots \mathbf{j}_{p}}(K)
$$

Since $\left|f_{\mathbf{i}_{1} \cdots \mathbf{i}_{p}}^{-1} f_{\mathbf{j}_{1} \cdots \mathbf{j}_{p}}(K)\right| \leq r^{-1}|K|$ and $f_{\mathbf{i}^{\prime}}^{-1}\left(K_{\mathbf{j}}\right) \subset B\left(0,2 r^{-1}|K|\right)$, it follows that

$$
f_{\mathbf{i}_{1} \cdots \mathbf{i}_{p}}^{-1} f_{\mathbf{j}_{1} \cdots \mathbf{j}_{p}}(K) \subset B\left(0,3 r^{-1}|K|\right) \subset B(0, R) .
$$

Thus $f_{\mathbf{i}^{\prime}}^{-1}\left(K_{\mathbf{j}}\right)$ is a subset of $F$ by the definition of $F$.

Now suppose there is an $\mathcal{E}$-connected family $\left\{K_{\mathbf{i}(j)}: j=1, \ldots, k\right\}$ in level $n$. Without loss of generality, we let $\left\{K_{\mathbf{i}^{(j)}}: j=1, \ldots, \ell(k)\right\}$ be the maximal subset of the $\mathcal{E}$-connected family such that any two consecutive elements meet and non-consecutive elements do not intersect. From Bandt's finite type condition, it is trivial to see that if $k$ tends to infinity then $\ell(k)$ also tends to infinity. Let $\mathbf{i}^{(1)}=\mathbf{i}_{1} \cdots \mathbf{i}_{n} \in \Lambda_{n}$. For $0 \leq p \leq n$, we define a set

$$
G_{p}:=\bigcup_{j=1}^{\ell(k)} f_{\mathbf{i}_{1} \cdots \mathbf{i}_{p}}^{-1}\left(K_{\mathbf{i}^{(j)}}\right) .
$$

Then one part of $G_{p}$, i.e., $f_{\mathbf{i}_{1} \cdots \mathbf{i}_{p}}^{-1}\left(K_{\mathbf{i}^{(1)}}\right)=K_{\mathbf{i}_{p+1} \cdots \mathbf{i}_{n}} \subset K \subset B(0,|K|)$. Especially, $G_{0}=\bigcup_{j=1}^{\ell(k)} K_{\mathbf{i}^{(j)}} \subset K \subset B(0,|K|)$. The diameter of $G_{p}$ is a monotonic increasing function about $p$. Moreover, if we take $\ell(k)$ large enough (i.e., $\ell(k)>N+1$ ) then $G_{n}$ will be not contained in $B(0, R)$ by the argument in the first paragraph. Hence we can choose a suitable $p$ such that $G_{p}$ is contained in the ball $B\left(0,2 r^{-1}|K|\right)$ but not contained in $B(0,2|K|)$. The first restriction guarantees $G_{p}$ is a subset of $F$ by the above claim. The second restriction implies there is a $\delta_{k}$-path in $F$ from $x$ to $y$, where $x \in K \subset B(0,|K|), y \notin B(0,2|K|)$ (see Figure 1) and $r^{n-p+1}|K| \leq \delta_{k} \leq r^{n-p-1}|K|$. Denote this $\delta_{k}$-path by $\Gamma_{k}$.

Next we argue that $n-p$ tends to infinity when $k$ goes to infinity. Otherwise, if $n-p$ is bounded by a number $q$, then the diameters of the sets $f_{\mathbf{i}_{1} \cdots \mathbf{i}_{p}}^{-1}\left(K_{\mathbf{i}^{(j)}}\right), 1 \leq j \leq$ $\ell(k)$ are larger than $r^{q+1}|K|$. This implies that $B(0, R)$ will contain arbitrarily many cylinders of size $r^{q+1}|K|$, which is impossible. 


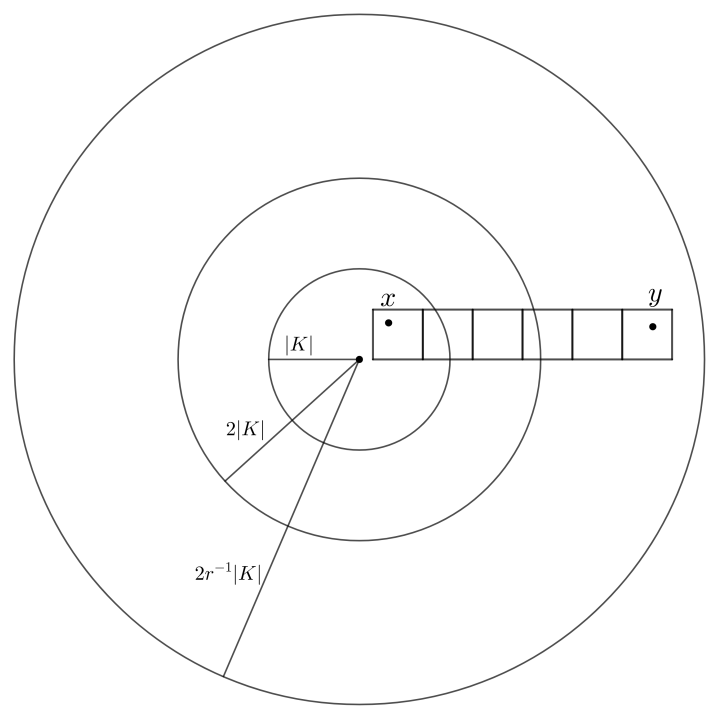

Figure 1.

Hence we have a sequence of $\delta_{k}$-paths $\left\{\Gamma_{k}\right\}$ in $F$, which starts from a point in $B(0,|K|)$ and ends at a point out of $B(0,2|K|)$. Let $\Gamma$ be a convergent point of $\Gamma_{k}$ under the Hausdorff metric $d_{H}[6]$. Then $\Gamma \subset F$ and $\Gamma$ is connected. Indeed, for any $x \in \Gamma$ and any $\epsilon>0$, the ball $B(x, \epsilon)$ must intersect some $\Gamma_{k}$ and hence $B(x, \epsilon) \cap F \neq \emptyset$. That is, $x$ is an accumulation point of $F$, and then $x \in F$ by the compactness of $F$. Secondly, if $\Gamma$ is not connected, then $\Gamma$ has a separation $\Gamma=A \cup B$ where $A$ and $B$ are nonempty closed sets and thus are compact sets, and

$$
\epsilon:=\inf \{|a-b|: a \in A, b \in B\}>0 .
$$

Let $\Gamma_{k}$ be a $\delta$-path such that $d_{H}\left(\Gamma_{k}, \Gamma\right)<\epsilon / 3$ and $\delta<\epsilon / 3$. Then $\Gamma_{k}$ is contained in an $\epsilon / 3$-neighborhood of $A$ and $B$, and $\Gamma_{k}$ cannot be a $\delta$-path. That completes the proof.

Acknowledgements. The authors would like to thank the referees very much for their many valuable comments and suggestions that make the paper more readable.

\section{References}

[1] Bandt, C., and M. Mesing: Self-affine fractals of finite type. - Banach Center Publications 84, 2009, 131-148.

[2] Bandt, C., and H. RAO: Topology and separation of self-similar fractals in the plane. Nonlinearity 20, 2007, 1463-1474.

[3] Bandt, C., and Y. Wang: Disk-like self-affine tiles in $\mathbf{R}^{2}$. - Discrete Comput. Geom. 26:4, 2001, 591-601.

[4] Deng, Q. R., K. S. LaU, and S. M. NGai: Separation conditions for iterated function systems with overlaps. - Contemp. Math. 600, 2013, 1-20.

[5] Hata, M.: On the structure of self-similar sets. - Japan J. Appl. Math. 2, 1985, 381-414.

[6] Hutchinson, J. E.: Fractals and self-similarity. - Indiana Univ. Math. J. 30, 1981, 713-747.

[7] Kenyon, R.: Self-replicating tilings. - In: Symbolic dynamics and its applications, Contemp. Math. 135, Amer. Math. Soc., Providence, RI, 1992, 239-263.

[8] Kirat, I., and K. S. LaU: On the connectedness of self-affine tiles. - J. London Math. Soc. 62, 2000, 291-304. 
[9] Lagarias, J. C.: Geometric models for quasicrystals I. Delone sets of finite type. - Discrete Comput. Geom. 2, 1999, 161-191.

[10] Lau, K. S., J. J. LuO, and H. RAO: Topological structure of fractal squares. - Math. Proc. Cambridge Philos. Soc. 155, 2013, 73-86.

[11] Leung, K. S., and K. S. LaU: Disk-likeness of planar self-affine tiles. - Trans. Amer. Math. Soc. $359,2007,3337-3355$.

[12] LeunG, K. S., and J. J. Luo: Connectedness of planar self-affine sets associated with nonconsecutive collinear digit sets. - J. Math. Anal. Appl. 395, 2012, 208-217.

[13] NGAI, S. M., and Y. WANG: Hausdorff dimension of self-similar sets with overlaps. - J. London Math. Soc. (2) 63:3, 2001, 655-672.

Received 27 May $2019 \bullet$ Accepted 26 April 2021 